\title{
Energy-time entanglement, Elements of Reality, and Local Realism
}

Jan-Åke Larsson

The self-archived postprint version of this journal article is available at Linköping University Institutional Repository (DiVA):

http://urn.kb.se/ resolve?urn=urn:nbn:se:liu:diva-58651

N.B.: When citing this work, cite the original publication.

Larsson, J., (2010), Energy-time entanglement, Elements of Reality, and Local Realism, QUANTUM THEORY: RECONSIDERATION OF FOUNDATIONS - 5, 1232, 115-127.

https:/ / doi.org/ 10.1063/ 1.3431481

Original publication available at:

https:// doi.org/ 10.1063/1.3431481

Copyright: AIP Publishing

http:// www.aip.org/ 


\section{Energy-time entanglement, Elements of Reality, and Local Realism}

Cite as: AIP Conference Proceedings 1232, 115 (2010); https://

doi.org/10.1063/1.3431481

Published Online: 03 May 2010

Jan-Åke Larsson

\section{ARTICLES YOU MAY BE INTERESTED IN}

Invited Review Article: Single-photon sources and detectors

Review of Scientific Instruments 82, 071101 (2011); https://

doi.org/10.1063/1.3610677

\section{Conference Proceedings}

\section{Get $30 \%$ off all print proceedings!}

Enter Promotion Code PDF30 at checkout 


\title{
Energy-time entanglement, Elements of Reality, and Local Realism
}

\author{
Jan-Åke Larsson \\ Department of Electrical Engineering and Department of Mathematics, \\ Linköpings Universitet, Linköping, Sweden
}

\begin{abstract}
This paper discusses energy-time entanglement experiments and their relation to Einstein-Podolsky-Rosen (EPR) elements of reality. The interferometric experiment proposed by J. D. Franson in 1989 provides the background, and the main issue here is a detailed discussion on whether a Local Realist model can give the Quantum-Mechanical predictions for this setup. The Franson interferometer gives the same interference pattern as the usual Bell experiment (modulo postselection). Even so, depending on the precise requirements made on the Local Realist model, this can imply a) no violation, b) smaller violation than usual, or c) full violation of the appropriate statistical bound. This paper discusses what requirements are necessary on the model to reach a violation, and the motivation for making these requirements. The alternatives include using a) only the measurement outcomes as EPR elements of reality, b) the emission time as EPR element of reality, and c) path realism. The subtleties of this discussion needs to be taken into account when designing and setting up future experiments of this kind, intended to test Local Realism.
\end{abstract}

Keywords: Non-locality, Bell inequality, Franson interferometer PACS: 03.65.Ud

\section{INTRODUCTION}

In 1989 a new experimental setup intended to test Local Realism was proposed by J. D. Franson [1]. The test in question needs postselection of events to violate the standard statistical bound for Local Realist models, the Bell inequality [2]. This need for postselection has been under discussion for some time, and the present note is intended to review the discussion and to provide some insight into the concepts and methods needed. Using these concepts, we will see that, depending on what is required from the tested model, the same experiment can give different results. Requiring Local Realism for the outcomes only does not in itself yield a violation, but requiring more of the model will yield a violation, which becomes stronger and stronger as the requirements on the model increases.

A central concept is "EPR elements of reality" as defined by Einstein, Podolsky, and Rosen (EPR) in 1935 [3]. The concept is well-known, but a brief repetition is in place. The setting is as follows: consider a (small) physical system on which we intend to measure position $Q$ and momentum $P$. The physical measurement devices associated with these measurements are mutually exclusive, and furthermore, the Quantum-Mechanical description for this physical system tells us that the measurements $Q$ and $P$ do not commute. The standard way to interpret this is that the system does not possess the properties of position or momentum, only probabilities are possible to obtain from Quantum Me- 
chanics.

What EPR ask in their paper is whether the Quantum-Mechanical description can be considered complete, or if it is possible to argue for another, more complete description. They use a combined system of two subsystems of the above type, in a combined state so that joint measurement of the positions gives the position mean $Q_{1}+Q_{2}=0$ and joint measurement of the two momenta give the momentum difference $P_{1}-P_{2}=0$. These two combinations are measurable at the same time, even though the individual positions and momenta are not, which means that it is possible to produce a joint state with these properties. Letting the two subsystems separate, usually very far, they consider individual measurement of position or momentum. The system is such that the position mean $\mathrm{V}$ and momentum difference is preserved under this process, which means that if the position of one subsystem has been measured, the position of the remote subsystem can be predicted. Therefore, EPR argue, the position of the remote subsystem must exist as a property of the subsystem. EPR write:

If, without in any way disturbing a system, we can predict with certainty (i.e., with a probability equal to unity) the value of a physical quantity, then there exists an element of physical reality corresponding to this physical quantity.

Likewise, if the momentum of one subsystem has been measured, the momentum of the remote subsystem can be predicted. In this case, the momentum of the remote subsystem must exist as a property of the subsystem. EPR continue to argue that both position and momentum must simultaneously exist as properties of the remote subsystem, otherwise

... the reality of $P$ and $Q$ depend[s] upon the process of measurement carried out on the first system, which does not disturb the second [remote] system in any way. No reasonable definition of reality could be expected to permit this.

The above definition of an "EPR element of reality" is the philosophical motivation for considering properties of a system as existing, independent of measurement. The possibility of remote prediction ("without in any way disturbing a system") enables the notion of "EPR element of reality," and we will use this notion below to motivate existence of properties of the involved physical systems.

The EPR setup is fine for philosophical considerations, but there are more powerful tools that enable a statistical test of experimental data. The setup to be used was proposed by D. Bohm in the fifties [4] (see Fig. 1), and uses a system combined of two spin-1/2 subsystems in a total spin 0 state, so that the spins $S_{1}+S_{2}=0$ when measured along

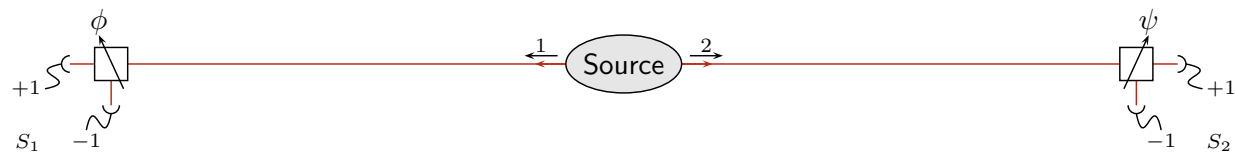

FIGURE 1. The EPR-Bohm-Bell setup. The two systems are spin-1/2 systems, and the local measurements are made along a direction in space $\phi$ or $\psi$, respectively. The source is such that if the directions $\phi=\psi$, the outcomes $S_{1}+S_{2}=0$ with probability one. 
equal directions $\phi=\psi$. The subsystems are allowed to separate and a spin measurement is made on one of the subsystems. The choice of measurement directions is a continuous choice instead of the dichotomic choice in the original EPR setup. When a measurement has been made on one subsystem, the result can be used to predict the result of a measurement on the remote subsystem along the same direction. Therefore, the spin along any direction is an EPR element of reality.

This was used in the celebrated Bell paper [2] where a statistical test was devised, an inequality that must be fulfilled by any mathematical model that is Realist and Local. Realism is here motivated by the spin being an EPR element of reality, and Locality is motivated by the finite speed of light, or more specifically, because local measurement is made "without in any way disturbing" the remote system (the below formulation is adapted from [5]).

Theorem 1:-A Local Realist model has the following two properties:

a) Realism. Outcomes are given by random variables (on a prob. space $(\Lambda, \mathscr{F}, P)$ ),

$$
S_{i}(\phi, \psi): \Lambda \mapsto\{ \pm 1\}
$$

b) Locality. Outcomes do not depend on the remote settings,

$$
S_{1}(\phi, \psi)=S_{1}(\phi), \quad S_{2}(\phi, \psi)=S_{2}(\psi) .
$$

The outcomes from a Local Realist model obey

$$
\left|E\left(S_{1}(\alpha) S_{2}(\delta)\right)+E\left(S_{1}(\alpha) S_{2}(\beta)\right)\right|+\left|E\left(S_{1}(\gamma) S_{2}(\beta)\right)-E\left(S_{1}(\gamma) S_{2}(\delta)\right)\right| \leq 2
$$

The last inequality is violated by the predictions of Quantum Mechanics, from which the correlation is obtained as

$$
\left\langle s_{1}(\phi) s_{2}(\psi)\right\rangle=-\cos (\phi-\psi)
$$

with $\phi-\psi$ being the angle between the two directions $\phi$ and $\psi$, and the minus sign is from using a total spin zero state. Choosing the four directions $\pi / 4$ apart in a plane in the order $\delta, \alpha, \beta, \gamma$, one obtains

$$
\left|\left\langle s_{1}(\alpha) s_{2}(\delta)\right\rangle+\left\langle s_{1}(\alpha) s_{2}(\beta)\right\rangle\right|+\left|\left\langle s_{1}(\gamma) s_{2}(\beta)\right\rangle-\left\langle s_{1}(\gamma) s_{2}(\delta)\right\rangle\right|=2 \sqrt{2}
$$

and this violates Ineq. (1). Therefore, the Quantum-Mechanical predictions cannot be obtained from a Local Realist model.

There is one problem that is present in most experiments done to test this inequality: inefficient detectors. This problem was first noticed by Pearle in 1970 [6], but has been treated in many subsequent papers. The problem is that one only obtains detections from a subset of the pairs in the experimental setup, the pairs that give coincident detections. The correlation obtained from experiment is conditioned on coincident detection of two particles, one at each side. Taking this into account, the theorem needs to be modified as follows (adapted from [5]). 
Theorem 2:-A Local Realist model with inefficiency has the following three properties:

a) Realism. Outcomes are given by random variables on subsets of detection (in a prob. space $(\Lambda, \mathscr{F}, P))$,

$$
S_{i}(\phi, \psi): \Lambda_{S_{i}, \phi, \psi} \mapsto\{ \pm 1\} .
$$

b) Locality. Outcomes and detections do not depend on the remote settings,

$$
S_{1}(\phi, \psi)=S_{1}(\phi) \text { on } \Lambda_{S_{1}, \phi, \psi}=\Lambda_{S_{1}, \phi}, \quad S_{2}(\phi, \psi)=S_{2}(\psi) \text { on } \Lambda_{S_{2}, \phi, \psi}=\Lambda_{S_{2}, \psi} .
$$

c) Efficiency. There is a lower bound to the efficiencies,

$$
\eta=\min _{\text {settings }} P(\text { coincidence } \mid \text { local detection })
$$

The outcomes from a Local Realist model with inefficiency obey

$$
\begin{aligned}
& \mid E\left(S_{1}(\alpha) S_{2}(\delta) \mid \text { coinc. }\right)+E\left(S_{1}(\alpha) S_{2}(\beta) \mid \text { coinc. }\right) \mid \\
& \quad+\mid E\left(S_{1}(\gamma) S_{2}(\beta) \mid \text { coinc. }\right)-E\left(S_{1}(\gamma) S_{2}(\delta) \mid \text { coinc. }\right) \mid \leq \frac{4}{\eta}-2 .
\end{aligned}
$$

The effect is that the inequality is weakened by inefficient detectors, and is no longer violated by Quantum Mechanics at $\eta=2 \sqrt{2}-2 \approx 82.83 \%$. We will see effects and extensions of this below.

\section{THE FRANSON INTERFEROMETER}

In 1989 a new experimental setup was proposed by J. Franson [1] (see Fig. 2). The setup uses a source that emits time-correlated photons at unknown moments in time, and two unbalanced Mach-Zehnder interferometers. The interferometers should have a path difference that is larger than the individual photon coherence time to prohibit first-order interference. Therefore, the probability is equal for a photon to emerge from each port of the final beamsplitter. But the interferometer path-differences should be as equal as possible; the path-difference difference (repetition intended) should be smaller than the coherence length of the photon pair. Since the emission time is unknown (and is a quantum variable in second quantization), there can be interference between two

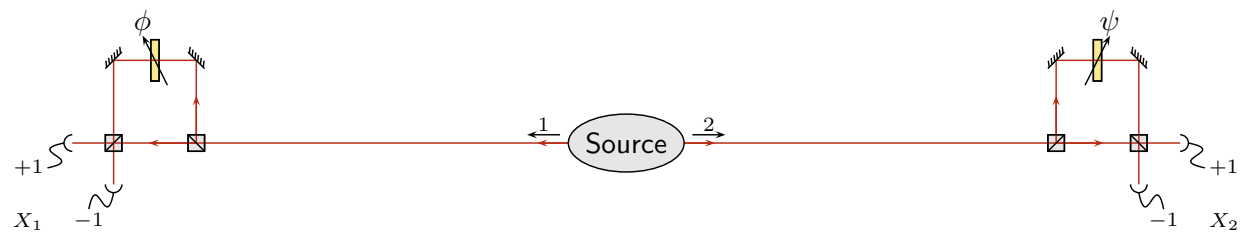

FIGURE 2. The Franson setup. The source sends out time-correlated photons at unknown moments in time. These travel through unbalanced (but equal) Mach-Zehnder interferometer with variable phase delays $\phi$ and $\psi$. If the detections are coincident and $\phi+\psi=0$, then $X_{1}=X_{2}$ with probability one. 
possiblities on the second beamsplitter: both photons "taking the long path" and both photons "taking the short path." Quotation marks are used here to remind the reader that photons are not particles, but quantum objects and as such, do not take a specific path.

There will be no interference if one photon "takes the long path" and the other "takes the short," because then, the emission time can be calculated easily as the early detection time minus the short path length divided by $c$. The emission time is known in this case. When both photons "take the same path," there is no way to calculate (or measure) the emission time, and this is what enables the interference. The interference is not visible as a change in output intensity, as in first-order interference, but instead in correlation of the outputs. Given coincident detection, if the total phase delay $\phi+\psi=0$, then a photon emerging in the +1 channel on the left is always accompanied by a photon emerging in the +1 channel on the right, and the same for the -1 channels. As a function of the total phase delay, we have

$$
\left.\left\langle x_{1}(\phi) x_{2}(\psi)\right| \text { coinc. }\right\rangle=\cos (\phi+\psi),
$$

note the similarity to Eq. (2). It is now simple to obtain

$$
\begin{aligned}
& \left.\left.\mid\left\langle x_{1}(\alpha) x_{2}(\delta)\right| \text { coinc. }\right\rangle+\left\langle x_{1}(\alpha) x_{2}(\beta)\right| \text { coinc. }\right\rangle \mid \\
& \left.\left.\quad+\mid\left\langle x_{1}(\gamma) x_{2}(\beta)\right| \text { coinc. }\right\rangle-\left\langle x_{1}(\gamma) x_{2}(\delta)\right| \text { coinc. }\right\rangle \mid=2 \sqrt{2},
\end{aligned}
$$

and from that obtain a violation of the bound in Ineq. (1). The important question is now: does this setup violate Local Realism?

The problem is that there is a Local Realist model that gives the exact same predictions as Quantum Mechanics [7] (see Fig. 3). Since the model is of the type described in Theorem 1, it is strange that it seems to violate Ineq. (1). It is important to note that the model does not violate the inequality; it is true that the model gives the correlation

$$
E\left(x_{1}(\phi) x_{2}(\psi) \mid \text { coinc. }\right)=\cos (\phi+\psi),
$$

but this is a conditional expectation, of the type used in Theorem 2. To reach this correlation we need to postselect data, and only use $50 \%$ of the events, so that $\eta=50 \%$,
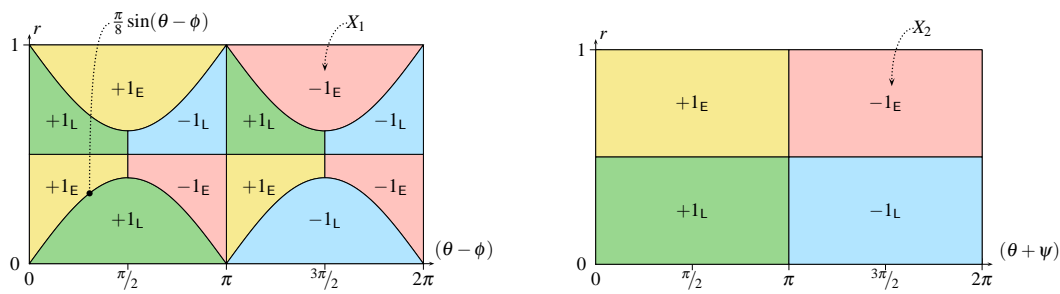

FIGURE 3. The model of [7]. The hidden variable is a pair of numbers $\lambda=(\theta, r)$ in the rectangle $\Lambda=\{(\theta, r): 0 \leq \theta<2 \pi, 0 \leq r<1\}$. The outcomes are determined by the above graphs where detections are "early" (E) or "late" (L). This model gives the quantum predictions for the Franson interferometric setup, including those for the coincident detections. 
well below the $82 \%$ bound where Quantum-Mechanical predictions are possible to reach with a Local Realist model.

Actually, this model uses a slightly different mechanism than inefficiency, it uses variable delays at the two sites, in which case the following theorem applies (for details see [8]).

Theorem 3:-A Local Realist model with delays has the properties a) and b) from Theorem 1, and

c) Delays. Detections occur after Local Realist time delays,

$$
T_{1}(\phi): \Lambda \mapsto \mathbb{R} \quad \text { and } \quad T_{2}(\psi): \Lambda \mapsto \mathbb{R}
$$

and usage of a coincidence window gives an apparent efficiency of

$$
\eta=\min _{\text {settings }} P(\text { coincidence } \mid \text { local detection })
$$

The outcomes from a Local Realist model with delays obey

$$
\begin{aligned}
& \mid E\left(X_{1}(\alpha) X_{2}(\delta) \mid \text { coinc. }\right)+E\left(X_{1}(\alpha) X_{2}(\beta) \mid \text { coinc. }\right) \mid \\
& \quad+\mid E\left(X_{1}(\gamma) X_{2}(\beta) \mid \text { coinc. }\right)-E\left(X_{1}(\gamma) X_{2}(\delta) \mid \text { coinc. }\right) \mid \leq \frac{6}{\eta}-4 .
\end{aligned}
$$

In the interferometric setup under study, the delays are not continuously distributed but rather limited to two values: "delayed" and "not delayed," and the coincidence window is chosen smaller than the delay so that a coincidence occurs only for equal delays on the two sides. In the above inequality, the bound for Quantum-Mechanical violation is $\eta=3-3 / \sqrt{2} \approx 87.87 \%$, which is higher than before. The apparent efficiency is still $50 \%$ which is far from the efficiency needed for a violation.

Of course it is desirable to re-establish a violation. To do this one must first understand the basic reason for the failure of Theorems 1-3 to provide a usable test, in other words, the basic reason for the existence of the model in Fig. 3. It is tempting to blame only the postselection, but this is not the whole story. The theorems treat the individual sites as black boxes; feed them a setting and they (may) give a (possibly delayed) \pm 1 outcome, much like the setup of Fig. 1. We will see that taking more properties of the setup into account will enable a violation, and the remainder of this paper will discuss the possible ways to do this.

\section{PATH REALISM}

The key ingredient of the Local Realist model above [7] is that the delay at one of the sites depend on the local setting on that side. One possibility is of course to require that the path taken by the photon is a realist property [9], and independent of the settings because of the order in which the optical elements occur in the path. In the above setup, a photon encounters the first beamsplitter before the variable phase delay, so it would therefore seem that the decision to take the long path or the short path must be independent of the phase delay setting. 
One should take some care here. This requires particle-like properties of the photons; a realist path taken by the photons. In itself, this does not prohibit interference. Ordinary one-particle interference is not prohibited because a particle-model could randomly select a path for the photon and send a "virtual" photon through the other path, and use the phase shift difference to determine through which output port to exit from the second beamsplitter. Two-particle interference in the present setup is also not prohibited by path realism itself, but path realism taken together with Local Realism will prohibit (large) interference. The reason for this is that the two together will reestablish the bound of Theorem 1, because that theorem will be valid separately both for undelayed and delayed coincidences.

Theorem 4:-A Local Realist model with path realism has the properties a) and b) from Theorem 1, and

c) Path realism. The path taken is a realist property, and is setting-independent. The outcomes from a Local Realist model with path realism obey

$$
\begin{aligned}
& \mid E\left(X_{1}(\alpha) X_{2}(\delta) \mid \text { coinc. }\right)+E\left(X_{1}(\alpha) X_{2}(\beta) \mid \text { coinc. }\right) \mid \\
& \quad+\mid E\left(X_{1}(\gamma) X_{2}(\beta) \mid \text { coinc. }\right)-E\left(X_{1}(\gamma) X_{2}(\delta) \mid \text { coinc. }\right) \mid \leq 2 .
\end{aligned}
$$

This means that postselection has no negative effects on the bound in a model with path realism, and the inequality is violated by the quantum prediction (5). Which means that there is no Local Realist model with path realism that gives the probabilities predicted by Quantum Mechanics.

It is important to note here that path realism is different from measurement-outcome realism. The outcomes are EPR elements of reality, because they are independent of what remote measurement was made. But above, the argument is that the "path taken by the photon" should be independent of the local measurement chosen. However, the path taken in this setup is not an EPR element of reality, because it cannot be remotely predicted. In fact, in the above setup, there is no measurement at one site that enables a path prediction for the remote site.

It is possible to dismantle the interferometer and measure which path the photon took in the interferometer, and predict from finding the photon in one path that it is not present in the other $[9,10]$. But that prohibits interference completely. It is central in the EPR reasoning that the system that we predict properties for, is unaffected by the measurement used for predictions. A path measurement removes interference, and in experimental setups of this kind experimenters go through great efforts to remove any possibility of extracting path information from the experiment precisely because of this. A path measurement would precede the interferometric measurement so locality cannot be used to argue independence of path prediction and an interferometric measurement. (Noncontextuality cannot be used either since the measurements do not commute.) The EPR argument does not support path realism because no prediction can be made "without in any way disturbing" the system.

This should not be read as an argument against testing path realism together with Local Realism using this setup. It is perfectly reasonable to list path realism as an expected model property, and to test it. Bringing in even more properties from classical physics into the picture, models like that in Fig. 3 can be argued to be inconsistent [9]. 
However, there is no doubt that an experiment like the above cannot be modeled within classical physics; that any model would yield an inconsistency with respect to classical physics, as noted in [9]. The present discussion on Local Realism is on a lower level, and attempts to determine exactly what requirements are needed to give a contradiction with quantum predictions. Bringing in all of classical physics would give an unwieldy result that would not tell us more about the nature of the world than we already know. It is important to pinpoint the exact place where a contradiction is reached, precisely which properties that in combination cannot be satisfied. To reestablish the bound (9), the properties needed are Local Realist outcomes (supported by the EPR argument) and realist photon path.

\section{LOCAL REALISM ONLY}

It is now interesting to ask whether it is possible to obtain a violation using only EPR elements of reality. Obviously, Local Realist measurement outcomes is not enough. It is possible to establish another EPR element of reality in this setup, but it is slightly different than the path realism used above. By removing the first beamsplitter at one site, the detection time can be used to calculate the time of emission of the photon pair. And this enables prediction of the emission moment for the remote photon, without in any way disturbing it. Therefore, the moment of emission is an EPR element of reality. Which means that a Local Realist model must be similar to the one in Fig. 3, in that it needs to specify the delay: whether a detection is "early" or "late." This is not the same as specifying the path because it does not require that the photon "takes a path," but only that the detection is delayed or not by the interferometric setup.

In this situation, the settings $\phi$ and $\psi$ can still influence whether a delay or not occurs because the detection takes place after the photon has (or could have) passed the phase delay. There are two possible detection moments, and the appropriate setting is the one that was set at the time when the photon passed (could have passed) the phase delay. This means that there are actually two settings that can influence the detection moment: one "early setting" for the early detection, and one "late setting" for the late detection (see Fig. 4).

The time between detection and the relevant setting is given by the path distance from the phase delays to the detectors, while the time between the early and the late detection is given by the path-length difference within one of the unbalanced interferometers. By putting the phase delay as close to the detectors as possible, and/or by using a suitably large path difference in the interferometers, it would be possible to ensure that the late setting occurs after the early detection has taken place, as hinted in Fig. 5.

If this is the case, the early setting can always influence the detection so that it is

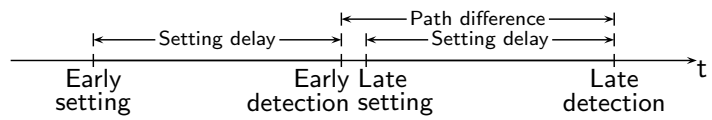

FIGURE 4. Two settings influence the outcome, one for the early detection, and one for the late detection 


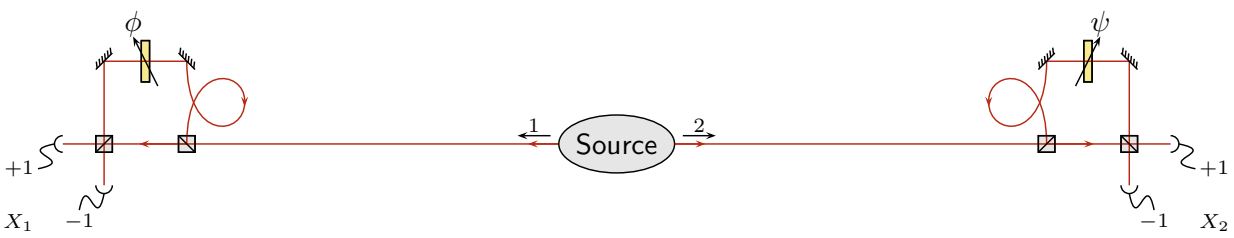

FIGURE 5. Ensuring that the setting appropriate for the late detection is input after the early detection would have taken place. This requires a phase delay close to the detectors, and/or a large path difference between the short and the long path.

delayed, but the late setting cannot undo the delay. This is because the early detection event would already have taken place when the late setting is input to the device, or even chosen. This means that, while there is no bound (except the trivial) for the outcomes in the early coincidences, Theorem 1 applies for the late coincidences and this gives the following theorem [7].

Theorem 5:-A Local Realist model with long realist delays has the properties a) and b) from Theorem 1, and

c) Time-delay realism. The delay is a realist property, and is long enough to ensure that the setting relevant for the late detection cannot undo the delay.

The outcomes from a Local Realist model with long realist delays obey

$$
\begin{aligned}
& \mid E\left(X_{1}(\alpha) X_{2}(\delta) \mid \text { coinc. }\right)+E\left(X_{1}(\alpha) X_{2}(\beta) \mid \text { coinc. }\right) \mid \\
& \quad+\mid E\left(X_{1}(\gamma) X_{2}(\beta) \mid \text { coinc. }\right)-E\left(X_{1}(\gamma) X_{2}(\delta) \mid \text { coinc. }\right) \mid \leq 3 .
\end{aligned}
$$

The bound in (10) is 3; this is the mean value of the trivial bound 4 for the early coincidences and the bound 2 for the late coincidences. Unfortunately, this is larger than the maximal quantum prediction $2 \sqrt{2}$. To establish a better bound we need to use socalled "chained" Bell inequalities [11] with more terms, and this gives the following theorem [7].

Theorem 6:-The outcomes from a Local Realist model with long realist delays (as specified in Theorem 5) obey

$$
\begin{aligned}
& \mid E\left(X_{1}(\alpha) X_{2}(\varphi) \mid \text { coinc. }\right)+E\left(X_{1}(\alpha) X_{2}(\beta) \mid \text { coinc. }\right) \mid \\
& \quad+\mid E\left(X_{1}(\gamma) X_{2}(\beta) \mid \text { coinc. }\right)+E\left(X_{1}(\gamma) X_{2}(\delta) \mid \text { coinc. }\right) \mid \\
& \quad+\mid E\left(X_{1}(\varepsilon) X_{2}(\delta) \mid \text { coinc. }\right)-E\left(X_{1}(\varepsilon) X_{2}(\varphi) \mid \text { coinc. }\right) \mid \leq 5 .
\end{aligned}
$$

Again the bound is the mean value of the trivial bound 6 for the early coincidences and the chained-Bell bound 4 for the late coincidences. While the postselection does have an effect on the bound, the Quantum-Mechanical prediction still gives a violation; choosing the six directions $\pi / 6$ apart in a plane in the order $\varphi, \alpha, \beta, \gamma, \delta$, $\varepsilon$, will yield 
the quantum prediction

$$
\begin{aligned}
& \left.\left.\mid\left\langle x_{1}(\alpha) x_{2}(\varphi)\right| \text { coinc. }\right\rangle+\left\langle x_{1}(\alpha) x_{2}(\beta)\right| \text { coinc. }\right\rangle \mid \\
& \left.\left.\quad+\mid\left\langle x_{1}(\gamma) x_{2}(\beta)\right| \text { coinc. }\right\rangle+\left\langle x_{1}(\gamma) x_{2}(\delta)\right| \text { coinc. }\right\rangle \mid \\
& \left.\left.\quad+\mid\left\langle x_{1}(\varepsilon) x_{2}(\delta)\right| \text { coinc. }\right\rangle-\left\langle x_{1}(\varepsilon) x_{2}(\varphi)\right| \text { coinc } .\right\rangle \mid=3 \sqrt{3} \approx 5.20,
\end{aligned}
$$

There is no Local Realist model with setting-independent delays that gives the probabilities predicted by Quantum Mechanics. An experiment to test this is more difficult than an experiment to test Theorem 4, since it is less resilient to noise because the quantum violation is lower. Another important thing is that the switching of the settings should be fast enough to ensure that a new random setting is chosen in-between the early detection event and the late setting readoff. But this is the price to pay for only using EPR elements of reality in the derivation of the bound.

\section{MODIFIED SETUPS}

Another approach to reach a violation of Local Realism from the Quantum-Mechanical predictions is to modify the setup. A number of alternatives exist, and we will here briefly go through three of these alternatives.

The first was proposed by Strekalov et al in 1996 [12] (see Fig. 6). This setup uses a polarization-entangled source and three polarizing beamsplitters at each site. The interference occurs at the third and last polarizing beamsplitter. In this setup, the path taken is an EPR element of reality because a polarization measurement (in place of one interferometer) can be used to remotely predict which path the remote photon is going to travel through. But this is not needed; because of the polarization entanglement, the photon pairs are either jointly delayed or not, so that every pair gives coincident detections. There is no postselection, and we can in fact use Theorem 1 (modulo other experimental problems). This is good, because the experimental realization proposed in [12] does not use separate paths, but instead uses a single birefringent optical element at each site to implement the whole unbalanced interferometer, so an argument based on a

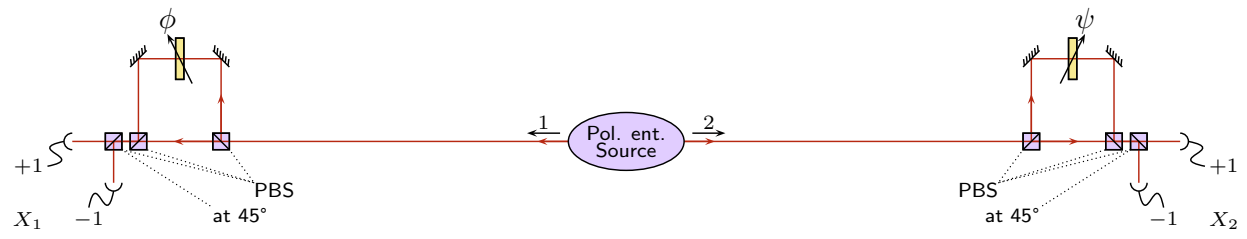

FIGURE 6. Using a polarization-entangled source. The time-correlated photons are still sent out at unknown moments in time, but are now also polarization-entangled. The beamsplitters used are polarizing beamsplitters (PBS), and the interference occurs at the third PBS at each site, because this is oriented $\pi / 4$ in relation to the other two. In this setup there is no postselection, so that Theorem 1 can be used directly, and the bound is violated by the quantum prediction. 


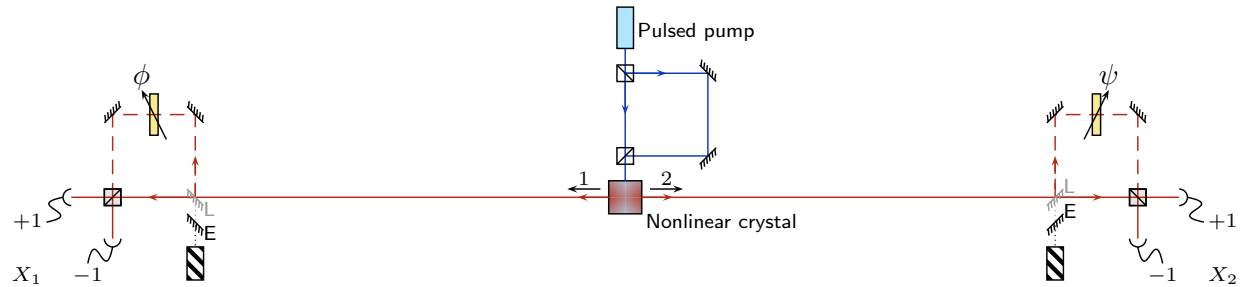

FIGURE 7. Using controlled mirrors. The mirrors are synchronized with the source so that photons produced by the early part of the pulse would be delayed in the interferometer, while photons produced by the late part of the pulse would not be delayed. Also in this setup there is no postselection, so that Theorem 1 can be used directly, and the bound is violated by the quantum prediction.

realist path cannot be used. But as we have seen, Local Realism can be violated in this setup even when the paths coincide.

The second alternative uses a source proposed in Brendel et al in 1999 [13] and switched mirrors in place of the first beamsplitters [14]. The source uses a pulsed pump, an unbalanced interferometer, and a nonlinear device that creates photon pairs. The nonlinear device [15] is often used in the previous setups as well, both with a pulsed and continuous pump, but here it is important that the source is pulsed. The active mirrors are pushed in and pulled out of the photon path in sync with the source (see Fig. 7). In this setup, the path taken is also an EPR element of reality, because a measurement of the time of emission (in place of one interferometer) can be used to remotely predict which path the remote photon is going to travel through. Again, this is not needed; because of the synchronization of the mirror positions, the photon pairs are either jointly delayed or not, and again every pair gives coincident detections. There is no postselection, and Theorem 1 (modulo other experimental problems) can be used to rule out Local Realist models.

The third alternative in this list was proposed by Cabello et al in 2009 and uses crosscoupled interferometers (see Fig. 8). In this setup, postselection is performed because the two photons may both end up at the same site, at both input ports of the beamsplitter at that site but delayed with respect to each other. The events that give coincident detection at both sites make up 50\% of the total. But also here the path is an EPR element of

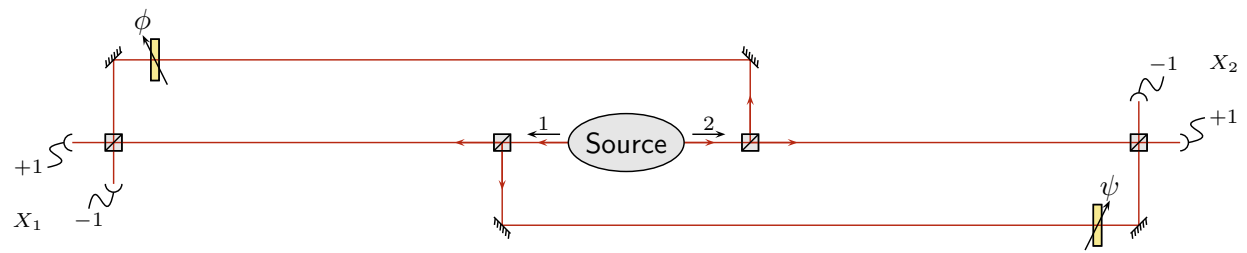

FIGURE 8. Cross-coupled interferometers. This setup has many of the properties of the original Franson setup. The main differences are that it is has very large interferometers (experimentally challenging), and that the path taken by each photon is an EPR element of reality, so that Theorem 4 can be used, and the bound is violated by the quantum prediction. 
reality, because a local path measurement (done by removing the final beamsplitter) can be used to remotely predict which path the remote photon is going to emerge from. Since the path is an EPR element of reality, we can use Theorem 4 (modulo other experimental problems), and the quantum prediction will violate the bound. There is no Local Realist model (with realist path) that gives the quantum predictions of this setup.

\section{CONCLUSIONS}

This paper has briefly discussed the basis of tests of Local Realism using energy-time entanglement. Even though most of the proposed experiments use postselection, we have seen that proper tests are possible. But we have also seen that some care needs to be taken because, depending on the precise requirements made on the Local Realist model, the same experiment result can imply a) no violation, b) smaller violation than usual, or c) full violation of the appropriate statistical bound:

a) Using only that the measurement outcomes are EPR elements of reality is not enough to yield a violation.

b) Adding that the moment of emission is an EPR element of reality enables a violation, although a weaker violation than that of the usual Bell setup.

c) Requiring that the model also uses a realist path will enable a violation, equally strong as that of the usual Bell setup.

Note that, in the original setup, the path is not an EPR element of reality; it cannot be predicted without disturbing the system. It is perfectly reasonable to list path realism as an expected model property, and to test it. One motive for requiring this particle-like behaviour from the model would be correspondence to properties from classical physics. An alternative is to modify the setup:

d) Ensuring that the path taken is an EPR element of reality by modifying the setup enables a violation, equally strong as that of the usual Bell setup.

Energy-time entanglement obtained with the Franson interferometer and its variants will continue to be used in experiments intended to violate Local Realism. It is important to be aware of exactly what is violated in these experiments, and the size of the violation. Even with the subtleties associated with this interferometer, or more correctly, because of these subtleties, the interferometer will continue to be an important tool to extend our knowledge on the foundations of Quantum Mechanics.

\section{ACKNOWLEDGMENTS}

I would like to acknowledge discussions with J. D. Franson and A. Cabello.

\section{REFERENCES}

1. J. D. Franson, Phys. Rev. Lett. 62, 2205-2208 (1989). 
2. J. S. Bell, Physics 1, 195-200 (1964).

3. A. Einstein, B. Podolsky, and N. Rosen, Phys. Rev. 47, 777-780 (1935).

4. D. Bohm, and Y. Aharonov, Phys. Rev. 108, 1070-1076 (1957).

5. J.-A. Larsson, Phys. Rev. A 57, 3304-3308 (1998).

6. P. M. Pearle, Phys. Rev. D 2, 1418-1425 (1970).

7. S. Aerts, P. Kwiat, J.-Å. Larsson, and M. Żukowski, Phys. Rev. Lett. 83, 2872-2875 (1999).

8. J.-Å. Larsson, and R. D. Gill, Europhys. Lett. 67, 707-713 (2004).

9. J. D. Franson, Phys. Rev. A 61, 012105-1-5 (2000).

10. J. D. Franson, Phys. Rev. A 80, 032119-1-9 (2009).

11. S. L. Braunstein, and C. M. Caves, Annals of Physics 202, 22-56 (1990).

12. D. V. Strekalov, T. B. Pittman, A. V. Sergienko, Y. H. Shih, and P. G. Kwiat, Phys. Rev. A 54, R1-R4 (1996).

13. J. Brendel, N. Gisin, W. Tittel, and H. Zbinden, Phys. Rev. Lett. 82, 2594-2597 (1999).

14. W. Tittel, J. Brendel, N. Gisin, and H. Zbinden, Phys. Rev. A 59, 4150-4163 (1999).

15. P. G. Kwiat, K. Mattle, H. Weinfurter, A. Zeilinger, A. V. Sergienko, and Y. Shih, Phys. Rev. Lett. 75 , 4337-4341 (1995). 